\title{
Linear Manifolds in Sets of Solutions of Quaternionic Polynomial Equations of Several Types
}

\author{
Dmytro Mierzejewski
}

\begin{abstract}
We give complete description of possible shapes of the set of the solutions of any quaternionic equation of the form $a x+x b=c$. Moreover we study the set of the solutions of a quaternionic equation of the form $a x^{2}+x^{2} b=c$ by the method of sections by hyperplanes perpendicular to the real axis; for every case where such section is an unbounded linear manifold a necessary and sufficient condition is found.
\end{abstract}

Mathematics Subject Classification (2010). 11R52.

Keywords. Quaternion, polynomial equation, linear manifold, system of equations, section by a hyperplane.

\section{Introduction}

Problems about zeros of quaternionic polynomials turn out to be much more difficult than the ones about zeros of real or complex polynomials. In particular, the non-commutativity of quaternions makes the general problem very complicated since the general form of an arbitrary quaternionic polynomial is

$$
\sum_{p=1}^{n}\left(\sum_{\ell=1}^{m_{p}} a^{(p, \ell, 1)} x a^{(p, \ell, 2)} x \ldots a^{(p, \ell, p)} x a^{(p, \ell, p+1)}\right)+c .
$$

It is effective to study different types of quaternionic polynomials separately. From old works on this topic we should notice [7] and [1]. In [7] it is described in detail how to extract all $n$th roots of a quaternion, that is, how to find all zeros of a quaternionic polynomial of the form $x^{n}-a$. In [1] it is proved that if a quaternionic polynomial has only one term of the highest degree then it has at least one quaternionic zero. (By the way, it is easy to show that in general this "fundamental theorem of algebra" is not true for quaternionic polynomials; for more detailed explanation see Remark 1 after the proof of Theorem 1.) 
During the last dozen of years the topic was intensively investigated by different mathematicians. The most successfully investigated quaternionic polynomials are the ones of the form

$$
\sum_{p=0}^{n} a^{(p)} x^{p}
$$

or

$$
\sum_{p=0}^{n} x^{p} a^{(p)} .
$$

We will call them classical ones (other authors use also other terms for such polynomials: simple, unilateral, regular). Note that, of course, a problem about zeros of a polynomial is equivalent to a problem about solutions of an equation, and we will use both these methods to describe problems. Thus we will use also the term "classical polynomial quaternionic equation" which means an equation of the form $f(x)=0$, where $f(x)$ is a classical quaternionic polynomial. Moreover we will use the term "non-classical" for quaternionic polynomials and polynomial quaternionic equations which are not classical.

A theory about all possible types of the sets of the zeros of the classical quaternionic polynomials is described, for example, in [9]. A convenient method to calculate zeros of certain such polynomials is described in [2] (p. 663-664); we would like to note also that in [2] only polynomials with right coefficients are considered, and for the ones with left coefficients it is necessary somewhat to change formulas from the just mentioned fragment of [2]. In [6] some additional investigations of quadratic classical quaternionic polynomials were fulfilled.

As for recent works on non-classical quaternionic polynomials, we can refer to papers [13], [3], [6], [4], [5], [8], [12], and also to the abstract [11] of a report at a scientific conference. It is interesting to note that often one manages to obtain essential results about non-classical quaternionic polynomials by only some kinds of "elementary" methods.

An important example of such method is the passage from a quaternionic equation to the corresponding system of four real equations with four unknowns, obtaining by direct calculations (that is, equalling coefficients at the same quaternionic units after the fulfilment of arithmetical operations with the unknown and all parameters written in the form $\xi_{0}+\xi_{1} i+\xi_{2} j+\xi_{3} k$ ). Just this method was used for investigations of linear quaternionic equations in [11] and [3]. For non-linear equations the method often leads to very complicated expressions, but it proved to be fruitful for investigations in some particular cases (see [4], [12], [5]) and seems to be necessary (though difficult) for further investigations of non-classical quaternionic equations.

It is interesting to note about one more feature of investigations performed in [4] and [5]. Investigated in those papers are in particular the sets of the solutions of certain types of quadratic quaternionic equations. Since these sets are situated in the four-dimensional space of the quaternions, it 
is convenient to study sections of the sets by three-dimensional hyperplanes (because for a human it is easier to imagine three-dimensional shape than four-dimensional one). In order to propose a proper family of hyperplanes it is possible, for example, to choose $q \in\{0,1,2,3\}$ and to consider sets of such solutions $x_{0}+x_{1} i+x_{2} j+x_{3} k$ which have the same fixed value of $x_{q}$. It turns out that the best way is to put $q=0$, that is, to consider sections by hyperplanes perpendicular to the real axis. Just such sections have been studied in [4] and [5].

It turns out that for many types of polynomial quaternionic equations the above mentioned sections are of the following shapes: a linear manifold, a sphere, a circle, the empty set, or a union of a finite number of sets each of them is one of the just mentioned shapes. In [5] any equation with this property is called multi-quasi-spherical (and there is also another term quasispherical for a certain narrower class of equations). Up to now it is unknown (at least for the author) any polynomial quaternionic equation which would be not multi-quasi-spherical. It is a reasonable hypothesis that every quadratic quaternionic equation or even every polynomial quaternionic equation is multi-quasi-spherical. ${ }^{1}$ (As for the linear ones, it is known that their sets of the solutions are always linear manifolds if not empty).

This paper is devoted to detailed investigations of possible shapes of the sets of the solutions of polynomial quaternionic equations of certain comparatively simple kinds, namely: a linear quaternionic equation $a x+x b=c$ and a quadratic one $a x^{2}+x^{2} b=c$. It is already known that both these equations are quasi-spherical (see [11], [5]). We obtain more detailed information by the method of the passage to a system of real equations. The equation $a x+x b=c$ was investigated also in Section 2 of [3] (where the authors called it Sylvester's one). We widen this information investigating possible ranks of the matrix of the corresponding system and making corresponding conclusions about the shapes. As for the equation $a x^{2}+x^{2} b=c$, we apply the above mentioned method of sections and investigate only cases of linear manifolds in these sections (according to the title of this paper).

Note also that information about each equation of the form $a x+x b=c$ or $a x^{2}+x^{2} b=c$ immediately implies information about each equation of the form $\alpha x \beta+\gamma x \delta=\lambda$ or $\alpha x^{2} \beta+\gamma x^{2} \delta=\lambda$ because it is easy to multiply the equations by $\beta^{-1}$ on the right and by $\gamma^{-1}$ on the left (of course, if $\beta, \gamma \neq 0$, but otherwise the equations are very simple).

\section{Preliminaries}

We use $\mathbb{H}$ as the standard notation for the set of all (real) quaternions. (We do not deal with the so-called complex quaternions whose components are complex). The notations $\mathbb{C}, \mathbb{R}$ and so on have their usual sense (the sets of, respectively, complex numbers, real numbers and so on).

\footnotetext{
${ }^{1}$ After submitting this paper the author has found out that the hypothesis is wrong: $i x i x+x i x i=0$ is a quadratic quaternionic equation being not multi-quasi-spherical.
} 
We use the standard notations $i, j, k$ for the quaternionic imaginary units; recall that $i^{2}=j^{2}=k^{2}=-1, \quad i j=-j i=k, \quad j k=-k j=i, \quad k i=$ $-i k=j$. We always use subindices to denote the components of a quaternion as follows: $\xi=\xi_{0}+\xi_{1} i+\xi_{2} j+\xi_{3} k$, where $\xi_{0}, \xi_{1}, \xi_{2}, \xi_{3} \in \mathbb{R}$; moreover $\xi_{0}$ is called the real part of $\xi$, and $\xi_{1} i+\xi_{2} j+\xi_{3} k$ is called its vector part. The modulus of a quaternion $\xi$ is for definition $|\xi|:=\sqrt{\xi_{0}^{2}+\xi_{1}^{2}+\xi_{2}^{2}+\xi_{3}^{2}}$.

We often treat sets of some quaternions geometrically using the wellknown interpretation of any quaternion $\xi_{0}+\xi_{1} i+\xi_{2} j+\xi_{3} k$ as the point $\left(\xi_{0}, \xi_{1}, \xi_{2}, \xi_{3}\right)$ of the four-dimensional space (here $\xi_{0}, \xi_{1}, \xi_{2}, \xi_{3} \in \mathbb{R}$ ).

In every equation of this paper the letter $x$ denotes the unknown and other letters denote given parameters, if there is no other explanation. We name a quaternionic equation any one in which every known parameter is a quaternion; as for solutions of such equations, we always consider solutions being quaternions. Analogously, real equations are the ones with real parameters and real solutions being considered.

\section{The Set of the Solutions of a Sylvester's Linear Quaternionic Equation}

In this section we will investigate quaternionic equations of the form

$$
a x+x b=c,
$$

which are sometimes referred to as Sylvester's ones.

Theorem 1. Let a quaternionic equation of the form (1) be given. Then the set of all quaternionic solutions of this equation is one of the following shapes:

1) the empty set;

2) one point;

3) a (straight) line;

4) a plane;

5) the set of all quaternions.

(We stress that we have listed here all possible kinds of linear manifolds excepting a (three-dimensional) hyperplane.) For each of these five shapes there exist such quaternions $a, b, c$ for which the set of the solutions of (1) is just of the given shape. The second kind of the shape occurs if and only if $b_{0} \neq-a_{0}$ or $|a| \neq|b|$. The fifth kind of the shape occurs if and only if $a, b \in \mathbb{R}, \quad b=-a, c=0$.

Proof. Firstly we will pass from (1) to the corresponding system of four real equations with four unknowns. For this aim we rewrite (1) as follows:

$$
\begin{aligned}
& \left(a_{0}+a_{1} i+a_{2} j+a_{3} k\right)\left(x_{0}+x_{1} i+x_{2} j+x_{3} k\right) \\
& +\left(x_{0}+x_{1} i+x_{2} j+x_{3} k\right)\left(b_{0}+b_{1} i+b_{2} j+b_{3} k\right)=c_{0}+c_{1} i+c_{2} j+c_{3} k .
\end{aligned}
$$


After simple transformations we get:

$$
\begin{aligned}
& \left(a_{0} x_{0}+b_{0} x_{0}-a_{1} x_{1}-b_{1} x_{1}-a_{2} x_{2}-b_{2} x_{2}-a_{3} x_{3}-b_{3} x_{3}\right) \\
& +\left(a_{1} x_{0}+b_{1} x_{0}+a_{0} x_{1}+b_{0} x_{1}-a_{3} x_{2}+b_{3} x_{2}+a_{2} x_{3}-b_{2} x_{3}\right) i \\
& +\left(a_{2} x_{0}+b_{2} x_{0}+a_{3} x_{1}-b_{3} x_{1}+a_{0} x_{2}+b_{0} x_{2}-a_{1} x_{3}+b_{1} x_{3}\right) j \\
& +\left(a_{3} x_{0}+b_{3} x_{0}-a_{2} x_{1}+b_{2} x_{1}+a_{1} x_{2}-b_{1} x_{2}+a_{0} x_{3}+b_{0} x_{3}\right) k \\
& =c_{0}+c_{1} i+c_{2} j+c_{3} k .
\end{aligned}
$$

Obviously, this equation is equivalent to the following system of four real equations with four unknowns:

$$
\left\{\begin{array}{l}
\left(a_{0}+b_{0}\right) x_{0}+\left(-a_{1}-b_{1}\right) x_{1}+\left(-a_{2}-b_{2}\right) x_{2}+\left(-a_{3}-b_{3}\right) x_{3}=c_{0} \\
\left(a_{1}+b_{1}\right) x_{0}+\left(a_{0}+b_{0}\right) x_{1}+\left(-a_{3}+b_{3}\right) x_{2}+\left(a_{2}-b_{2}\right) x_{3}=c_{1} \\
\left(a_{2}+b_{2}\right) x_{0}+\left(a_{3}-b_{3}\right) x_{1}+\left(a_{0}+b_{0}\right) x_{2}+\left(-a_{1}+b_{1}\right) x_{3}=c_{2} \\
\left(a_{3}+b_{3}\right) x_{0}+\left(-a_{2}+b_{2}\right) x_{1}+\left(a_{1}-b_{1}\right) x_{2}+\left(a_{0}+b_{0}\right) x_{3}=c_{3} .
\end{array}\right.
$$

Since the system (2) is linear, the set of the solutions is a linear manifold or the empty set. Of course, this fact is well-known for any linear quaternionic equation and was discussed in [11] and [3]. But to prove the theorem we have to show that: 1) the case of a hyperplane is impossible; 2) every other case is possible; 3) the last two sentences of the theorem are true; but the first of them in fact was proved in [3], so that only the second one remains to be proved.

Obviously, the set of all quaternions is the set of the solutions if and only if every coefficient of (2) equals zero, that is:

$$
\begin{aligned}
a_{0}+b_{0}=a_{1}+b_{1}=a_{2}+b_{2}=a_{3}+b_{3}=a_{1}-b_{1} & =a_{2}-b_{2}=a_{3}-b_{3} \\
& =c_{0}=c_{1}=c_{2}=c_{3}=0 .
\end{aligned}
$$

It is easy to see that it is the same that $a, b \in \mathbb{R}, b=-a, c=0$. So, the last sentence of the theorem is proved, and simultaneously it is proved that the 5 th kind of the shape occurs under some choice of $a, b, c$. It is also understandable at once that putting $a, b \in \mathbb{R}, \quad b=-a, c \neq 0$ one obtains the 1st kind of shape, that is the empty set.

In order to investigate the possibilities of the other linear manifolds it is enough to investigate possible ranks of the matrix of (2), that is

$$
\left(\begin{array}{cccc}
a_{0}+b_{0} & -a_{1}-b_{1} & -a_{2}-b_{2} & -a_{3}-b_{3} \\
a_{1}+b_{1} & a_{0}+b_{0} & -a_{3}+b_{3} & a_{2}-b_{2} \\
a_{2}+b_{2} & a_{3}-b_{3} & a_{0}+b_{0} & -a_{1}+b_{1} \\
a_{3}+b_{3} & -a_{2}+b_{2} & a_{1}-b_{1} & a_{0}+b_{0}
\end{array}\right)
$$

The rank 1 corresponds to a hyperplane, the rank 2 corresponds to a plane, and the rank 3 corresponds to a line (of course, for a proper value of $c$; by the way, $c=0$ is always proper). (The rank 4 corresponding to a point is not necessarily to be considered because the possibility of this case follows from the last but one sentence of the theorem, which was already discussed above.) 
Given (1) of the form $i x+x j=0$ we obtain (3) of the form

$$
\left(\begin{array}{cccc}
0 & -1 & -1 & 0 \\
1 & 0 & 0 & 1 \\
1 & 0 & 0 & -1 \\
0 & 1 & 1 & 0
\end{array}\right)
$$

whose rank is 3 . So, it is proved that a line occurs under some choice of $a$, $b, c$.

Then, given (1) of the form $(j+k) x+x(i+k)=0$ we obtain (3) of the form

$$
\left(\begin{array}{cccc}
0 & -1 & -1 & -2 \\
1 & 0 & 0 & 1 \\
1 & 0 & 0 & 1 \\
2 & -1 & -1 & 0
\end{array}\right)
$$

whose rank is 2 . So, it is proved that a plane occurs under some choice of $a$, $b, c$.

Now we have only to prove that the rank 1 is impossible for any matrix of the form (3). Suppose that (3) is of the rank 1 . Then any two columns of the matrix are proportional. Applying this information to the first two columns and considering the first two elements of each of them we can write the following proportion:

$$
\frac{a_{0}+b_{0}}{-a_{1}-b_{1}}=\frac{a_{1}+b_{1}}{a_{0}+b_{0}} .
$$

It is easy to see that this equality is impossible for real numbers if one treats it strictly; the only possibility for the proportionality to hold true is the presence of zeros, namely:

$$
a_{0}+b_{0}=a_{1}+b_{1}=0 .
$$

Then analogously we consider the first and third elements of the first and third columns, then the first and fourth elements of the first and fourth ones, and as a result we get:

$$
a_{0}+b_{0}=a_{1}+b_{1}=a_{2}+b_{2}=a_{3}+b_{3}=0 .
$$

Therefore now (3) is of the following form:

$$
\left(\begin{array}{cccc}
0 & 0 & 0 & 0 \\
0 & 0 & -a_{3}+b_{3} & a_{2}-b_{2} \\
0 & a_{3}-b_{3} & 0 & -a_{1}+b_{1} \\
0 & -a_{2}+b_{2} & a_{1}-b_{1} & 0
\end{array}\right)
$$

Taking into account the proportionality of the second and third columns of (4) it is easy to see that $a_{3}-b_{3}=0$. Then from analogous simple considerations it is easy to conclude that $a_{1}-b_{1}=a_{2}-b_{2}=0$. Thus we have concluded that every element of the matrix equals 0 , so that the rank is 0 that is in contradiction with the assumption. 
Remark 1. Though we proved occurring of the empty set by a very trivial example with zero coefficients, it is clear that non-trivial cases of the absence of a solution also occur. An example is $a x-x a=1$ for any $a \in \mathbb{H}$; this example (even in a more general form) was briefly discussed in [8] where the author wrote that he had taken it from [10]. Moreover it is not difficult to think up many other sufficiently different examples, considering certain matrices of the form (3) with the rank 2 or 3 and choosing a quaternion $c$ ensuring the absence of any solution.

\section{Unbounded Linear Manifolds in Sections of the Set of the Solutions of a Quaternionic Equation of the Form} $a x^{2}+x^{2} b=c$

Now we pass to the investigation of the following quaternionic equation:

$$
a x^{2}+x^{2} b=c .
$$

Note firstly that putting $x^{2}=: y$ one obtains a Sylvester's linear quaternionic equation. Such operation is probably reasonable if one tries to solve a certain equation of the form (5). But for general investigations of the shape of the sets of the solutions this is rather not effective since after finding $x$ by $y$ it is difficult to understand which shape will be constituted by all such $x$, even if the shape constituted by all $y$ is known. Therefore we will investigate this kind of equations by immediate passage to a system of real equations. By the way, in Section 3 of [5] the same method was applied for a wider class of equations, and thus we know from [5] that (5) is a quasi-spherical equation, according to the terminology of that paper.

Direct calculations give the following system equivalent to (5) (for the convenience of the further investigations we at once arrange addends in a way convenient to treat $x_{0}$ as a known number):

$$
\left\{\begin{array}{l}
\left(a_{0}+b_{0}\right)\left(x_{1}^{2}+x_{2}^{2}+x_{3}^{2}\right) \\
+2 x_{0}\left(\left(a_{1}+b_{1}\right) x_{1}+\left(a_{2}+b_{2}\right) x_{2}+\left(a_{3}+b_{3}\right) x_{3}\right)=\left(a_{0}+b_{0}\right) x_{0}^{2}-c_{0}, \\
\left(a_{1}+b_{1}\right)\left(x_{1}^{2}+x_{2}^{2}+x_{3}^{2}\right) \\
+2 x_{0}\left(-\left(a_{0}+b_{0}\right) x_{1}+\left(a_{3}-b_{3}\right) x_{2}+\left(b_{2}-a_{2}\right) x_{3}\right)=\left(a_{1}+b_{1}\right) x_{0}^{2}-c_{1}, \\
\left(a_{2}+b_{2}\right)\left(x_{1}^{2}+x_{2}^{2}+x_{3}^{2}\right) \\
+2 x_{0}\left(\left(b_{3}-a_{3}\right) x_{1}-\left(a_{0}+b_{0}\right) x_{2}+\left(a_{1}-b_{1}\right) x_{3}\right)=\left(a_{2}+b_{2}\right) x_{0}^{2}-c_{2}, \\
\left(a_{3}+b_{3}\right)\left(x_{1}^{2}+x_{2}^{2}+x_{3}^{2}\right) \\
+2 x_{0}\left(\left(a_{2}-b_{2}\right) x_{1}+\left(b_{1}-a_{1}\right) x_{2}-\left(a_{0}+b_{0}\right) x_{3}\right)=\left(a_{3}+b_{3}\right) x_{0}^{2}-c_{3} .
\end{array}\right.
$$

Similarly to [4] and [5], we will study the shape of the set of the solutions by the method of sections by hyperplanes perpendicular to the real axis. It means that we will treat $x_{0}$ as a fixed number and investigate which shape is determined by (6) in the three-dimensional space of points $\left(x_{1}, x_{2}, x_{3}\right)$.

So, let $x_{0}$ be fixed. Then it is obvious (and also known from [5]) that each equation from (5) determines a sphere, a linear manifold, or the empty set. We are interested in the intersection of the four figures determined by 
all the equations. Of course, this intersection may be a sphere, a circle, a set of two points, a linear manifold, or the empty set. The aim of Section 4 is to investigate carefully only cases where this intersection is an unbounded linear manifold, that is, a line, a plane, or the hyperplane.

Proposition 1. If the system (6) (with a fixed $x_{0}$ ) generates an unbounded linear manifold, then $b=-a$.

Proof. Obviously, any unbounded figure is impossible if at least one equation from (6) generates a bounded figure. But it is well-known that if the coefficient at the squares does not equal 0 then such equation generates a bounded figure (namely, a sphere, a point, or the empty set). Thus for an unbounded manifold to be the intersection it is necessary $a_{0}+b_{0}=a_{1}+b_{1}=a_{2}+b_{2}=a_{3}+b_{3}=0$, but it is the same that $b=-a$.

So, in what follows we will consider quaternionic equations only of the form

$$
a x^{2}-x^{2} a=c .
$$

Then we can rewrite (6) as follows:

$$
\left\{\begin{array}{l}
0=c_{0}, \\
4 x_{0}\left(a_{3} x_{2}-a_{2} x_{3}\right)=-c_{1}, \\
4 x_{0}\left(-a_{3} x_{1}+a_{1} x_{3}\right)=-c_{2}, \\
4 x_{0}\left(a_{2} x_{1}-a_{1} x_{2}\right)=-c_{3} .
\end{array}\right.
$$

Due to the first equation we conclude that the intersection is the empty set if $c_{0} \neq 0$. Thus looking for unbounded linear manifolds we can consider that $c_{0}=0$ and rewrite (7) as follows:

$$
a x^{2}-x^{2} a=c_{1} i+c_{2} j+c_{3} k .
$$

Moreover we can rewrite (8) without the first equation:

$$
\left\{\begin{array}{l}
4 x_{0}\left(a_{3} x_{2}-a_{2} x_{3}\right)=-c_{1}, \\
4 x_{0}\left(-a_{3} x_{1}+a_{1} x_{3}\right)=-c_{2}, \\
4 x_{0}\left(a_{2} x_{1}-a_{1} x_{2}\right)=-c_{3} .
\end{array}\right.
$$

Let us investigate firstly when (9) determines the whole hyperplane. It is easy to see that it occurs if and only if $c=0$ and either $x_{0}=0$, or $a_{1}=a_{2}=a_{3}=0$.

Note that in the case where $a_{1}=a_{2}=a_{3}=0$ (that is, $a \in \mathbb{R}$ ) the result does not depend on $x_{0}$, and thus in this case the set of the solutions is the whole set $\mathbb{H}$. This case is very trivial since it means that (5) has the form $\rho x^{2}-x^{2} \rho=0$ with $\rho \in \mathbb{R}$, so that it can be simply reduced to $0=0$. From the above considerations it is easy to see that in any other case it is impossible for all quaternions to be solutions of (5).

So, we have proved the following proposition:

Proposition 2. Let an equation of the form (5) be given. And let a real number $\xi_{0}$ be given. Then every quaternion whose real part equals $\xi_{0}$ is a solution of the equation if and only if the following conditions hold true: 
1) $b=-a$;

2) $c=0$;

3) $a \in \mathbb{R}$ or $\xi_{0}=0$.

Moreover the set of the solutions of the equation is the whole $\mathbb{H}$ if and only if the following conditions hold true:

1) $b=-a$;

2) $c=0$;

3) $a \in \mathbb{R}$.

Then we pass to the next kind of an unbounded linear manifold: a plane. Let us write down the matrix of the system (9):

$$
\left(\begin{array}{ccc}
0 & 4 x_{0} a_{3} & -4 x_{0} a_{2} \\
-4 x_{0} a_{3} & 0 & 4 x_{0} a_{1} \\
4 x_{0} a_{2} & -4 x_{0} a_{1} & 0
\end{array}\right) .
$$

For the case of the plane it is necessary for (10) to be of the rank 1 . But it is not difficult to see that such rank is impossible for such matrix. Really, supposing that the first column constitutes a basis of the set of the columns of (10) we see that there are only zeros in the first row; it means that $x_{0} a_{2}=$ $x_{0} a_{3}=0$, but then the first column also consists of only zeros and cannot constitute any basis. Analogously it is easy to refute each assumption that any other column constitutes a basis. So, it is proved the following:

Proposition 3. Let an equation of the form (5) be given. And let a real number $\xi_{0}$ be given. Consider the set of all such solutions of (5) whose real part equals $\xi_{0}$. Then this set is not a plane.

Let us look again at the matrix (10). Its determinant equals 0 . Therefore, since its rank cannot be 1 , we conclude that it is 0 or 2 . The case of the rank 0 had been considered earlier since it is the case of the hyperplane in the section (excepting the condition $c=0$ ). Thus the case of the rank 2 can be described as the negation of that case of the rank 0 (but under the conditions providing such form of the matrix), namely:

1) $b=-a$;

2) $c_{0}=0$

3) $a \notin \mathbb{R}$;

4) $\xi_{0} \neq 0$

Just this case of the rank 2 is the case of a line in the section if $c$ is proper (namely, $c_{0}=0$ and the column $\left(\begin{array}{l}c_{1} \\ c_{2} \\ c_{3}\end{array}\right)$ equals a linear combination of the columns of matrix (10); of course, to be precise one should change $x_{0}$ by $\xi_{0}$ in (10)). So, the following proposition is proved:

Proposition 4. Let an equation of the form (5) be given. And let a real number $\xi_{0}$ be given. Consider the set of all such solutions of (5) whose real part equals $\xi_{0}$. Then this set is a (straight) line if and only if the following conditions hold true: 
1) $b=-a$

2) $c_{0}=0$

3) $a \notin \mathbb{R}$;

4) $\xi_{0} \neq 0$;

5) the column $\left(\begin{array}{l}c_{1} \\ c_{2} \\ c_{3}\end{array}\right)$ equals a linear combination of the columns of the matrix

$$
\left(\begin{array}{ccc}
0 & 4 \xi_{0} a_{3} & -4 \xi_{0} a_{2} \\
-4 \xi_{0} a_{3} & 0 & 4 \xi_{0} a_{1} \\
4 \xi_{0} a_{2} & -4 \xi_{0} a_{1} & 0
\end{array}\right)
$$

So, we have investigated completely all the cases of unbounded linear manifolds in the sections. It is interesting also to note that the case where the section is one point (that is, a bounded linear manifold) is impossible if $b=-a$ (though it may occur if $b \neq-a$ ). This fact follows from the above observation that the rank of the matrix (10) cannot equal 3 (due to its determinant being equal 0 ).

Now we formulate a theorem created by accumulation information obtained in this section (see propositions and sentences emphasized by italics):

Theorem 2. Let an equation of the form (5) be given. And let a real number $\xi_{0}$ be given. Consider the set $S_{\xi_{0}}$ of all such solutions of (5) whose real part equals $\xi_{0}$. Then $S_{\xi_{0}}$ is one of the following types of shape:

1) the empty set;

2) a linear manifold;

3) two points;

4) a circle;

5) a sphere.

If $S_{\xi_{0}}$ is an unbounded linear manifold, then the following two conditions hold true:

1) $b=-a$;

2) $c_{0}=0$.

In any case $S_{\xi_{0}}$ is not a plane. If $S_{\xi_{0}}$ is one point, then $b \neq-a$.

$S_{\xi_{0}}$ is a (straight) line if and only if the following conditions hold true:

1) $b=-a$;

2) $c_{0}=0$;

3) $a \notin \mathbb{R}$;

4) $\xi_{0} \neq 0$;

5) the column $\left(\begin{array}{l}c_{1} \\ c_{2} \\ c_{3}\end{array}\right)$ equals a linear combination of the columns of the matrix (11).

$S_{\xi_{0}}$ is the whole hyperplane $x_{0}=\xi_{0}$ if and only if the following conditions hold true:

1) $b=-a$; 
2) $c=0$;

3) $a \in \mathbb{R}$ or $\xi_{0}=0$.

Moreover the set of the solutions of equation (5) is the whole $\mathbb{H}$ if and only if the following conditions hold true:

1) $b=-a$;

2) $c=0$;

3) $a \in \mathbb{R}$.

\section{References}

[1] S. Eilenberg, I. Niven, The "fundamental theorem of algebra" for quaternions. Bulletin of the American Mathematical Society vol. 50 no. 4 (1944), pp. 246248.

[2] G. Gentili, C. Stoppato, Zeros of regular functions and polynomials of a quaternionic variable. Michigan Math. Journal vol. 56 (2008), pp. 655-667.

[3] D. Janovská, G. Opfer, Linear equations in quaternionic variables. Mitt. Math. Ges. Hamburg vol. 27 (2008), pp. 223-234.

[4] D. Mierzejewski, Investigation of quaternionic quadratic equations I. Factorization and passing to a system of real equations. Bulletin de la Société des Sciences et des Lettres de Eódź. Série: Recherches sur les Déformations vol. 56 (2008), pp. 17-26.

[5] D. Mierzejewski, Quasi-spherical and multi-quasi-spherical polynomial quaternionic equations: introduction of the notions and some examples. Adv. appl. Clifford alg., this volume.

[6] D. Mierzejewski, V. Szpakowski, On solutions of some types of quaternionic quadratic equations. Bulletin de la Société des Sciences et des Lettres de Łódź. Série: Recherches sur les Déformations vol. 55 (2008), pp. 49-58.

[7] I. Niven, The roots of a quaternion. American Mathematical Monthly vol. 49 (1942), pp. 386-388.

[8] G. Opfer, Polynomials and Vandermonde matrices over the field of quaternions. Electronic Transactions on Numerical Analysis vol. 36 (2009), pp. 9-16.

[9] A. Pogorui, M. Shapiro, On the structure of the set of the zeros of quaternionic polynomials. Complex Variables and Elliptic Equations vol. 49 no. 6 (2004), pp. 379-389.

[10] S. Pumplün, S. Walcher, On the zeros of polynomials over quaternions. Comm. Algebra vol. 30 (2002), pp. 4007-4018.

[11] V. Szpakowski, Solution of general linear quaternionic equations. The XI Kravchuk International Scientific Conference. - Kyiv (Kiev), Ukraine, 2006, p. 661. (In Ukrainian.)

[12] V. Szpakowski, Solution of quadratic quaternionic equations. Bulletin de la Société des Sciences et des Lettres de Łódź. Série: Recherches sur les Déformations vol. 58 (2009) pp. 45-58.

[13] Y. Tian, Similarity and consimilarity of elements in the real Cayley-Dickson algebras. Adv. appl. Clifford alg. vol. 9 no. 1 (1999), pp. 61-76. 
Dmytro Mierzejewski

Teatralna Street, 5-b, ap. 6

Zhytomyr, 10014

Ukraine

e-mail: dmytro1972@gmail.com

Received: October 7, 2009.

Accepted: December 10, 2009.

Open Access This article is distributed under the terms of the Creative Commons Attribution Noncommercial License which permits any noncommercial use, distribution, and reproduction in any medium, provided the original author(s) and source are credited. 